\title{
XVe Congrès de la Société d'Andrologie de Langue Française
}

\section{LYON 10 - 11 - 12 Décembre 1998}

Hôtel Sofitel Lyon-Bellecour 20, Quai Qailleton 69002 Lyon

\section{JEUDI 11 Décembre}

8h30-9h30 Accueil-Café

9h30-9h40 Ouverture du Congrès

\section{GENETIQUE}

9h40-10h 10

10h $10-10 \mathrm{~h} 40$

10h40-11h
Génétique des infertilités

Microdélétions du chromosome $\mathrm{Y}$

Pause café

\section{CRYPTORCHIDIES}

11h -11h30 Aspects clinicobiologiques des cryptorchidies

11h30-12h Cryptorchidies : génétique moléculaire

12h $-12 \mathrm{~h} 30 \quad$ Cryptorchidies et impact des perturbateurs endocriniens présents dans l'environnement

12h30-14h Déjeuner

14h-16h30

\section{Séance posters et pause café}

\section{PLASMA SEMINAL}

16h30-17h Intérêt du plasma séminal pour le clinicien

J.ROLLET (France)

17h-17h30 Nouveaux marqueurs dans le plasma séminal pour l'étude des stérilités?

S.HAMAMAH (France)

17h30-18h L'apport de l'imagerie des glandes annexes J.F.LAPRAY (France)
B.SELE (France)

Ph.BERTA (France) 


\section{VENDREDI 12 Décembre}

\section{BIOPSIES TESTICULAIRES}

9h-9h30 Vascularisation du testicule humain : données récentes

9h30-10h Biopsies testiculaires dans les azoospermies non obstructives

10h-10h30 ISCI dans les azoospermies non obstructives : état des lieux

TABLE RONDE

11h-12h30 Les limites de l'AMP

12h30-14h Déjeuner

14h-15h30 Présentations orales

15h30-16h Pause café

\section{LES GENES DE LA SPERMATOGENESE}

16h-16h30 Apports des modèles de la transgenèse dans les physiopathologies des stérilités

D. DE ROOIJ (Pays-Bas)

16h30-17h Anomalies des gènes des récepteurs hormonaux dans les stérilités

I. HUHTANIEMI (Finlande)

17h -17h30 Clonage des gènes testiculaires humains : intérêt pour l'étude des stérilités

G.GUELLAEN (France)

20h Soirée de gala

\section{SAMEDI 12 Décembre}

\section{PHARMACOLOGIE DE LA SEXUALITE}

9h-9h30 Pharmacologie périphérique de l'éjaculation

9h30-10h Pharmacologie centrale de l'éjaculation

10h-10h30 Pause café

10h30-11h Pharmacologie périphérique de l'érection

11h-11h30 Pharmacologie centrale de l'érection

$11 \mathrm{~h} 30-12 \mathrm{~h} \quad$ Inhibiteurs de recapture de la sérotonine et sexualité

$12 \mathrm{~h}$

\section{Buffet de clôture}

S.ERGUEN (Allemagne)

P.SCHLEGEL (USA)

R.SCHOYSMAN (Belgique) 


\section{COMMUNICATIONS ORALES ET AFFICHEES}

Votre résumé devant figurer dans le livre du congrès, et étant imprimé suivant votre original, nous vous demandons de vous conformer strictement aux recommandations ci-après :

- Le résumé doit être en français

- Comporter le titre, les auteurs, l'adresse, le texte de la communication

- Etre dactylographié sur un format A4 dans un cadre de $22 \mathrm{~cm}$ de hauteur par $16 \mathrm{~cm}$ de largeur

- Etre présenté en simple interligne, police Times 12 points.

Un original et 4 copies doivent être envoyés avant le ler octobre 1998 à l'organisation du congrès :

Dr. M. BENAHMED - CONGRES «SALF 98»

INSERM Unité 407 - Faculté de Médecine Lyon-Sud - BP 12 F-69921 Oullins Cedex

Le Comité Scientifique sélectionnera les communications soit pour une présentation orale, soit pour une présentation affichée. La notification aux auteurs sera faite avant le ler novembre 1998.

- Les communications orales seront de 10 minutes, en français, suivie de 5 minutes de discussion.

Les voyages \& inscriptions des conférenciers des communications orales seront pris en charge par la SALF.

- Les communications affichées seront en français, sur poster de $90 \mathrm{~cm}$ de large par $120 \mathrm{~cm}$ de haut. Un prix récompensera la meilleure communication affichée.

\section{INFORMATIONS GENERALES}

\section{RENSEIGNEMENTS - INSCRIPTIONS}

Mohamed BENAHMED - Geneviève CECILLON

INSERM Unité 407 - Faculté de Médecine Lyon-Sud. BP 1269921 Oullins Cedex - France

Téléphone : 0478861606 - Fax: 0478865922

\section{LIEU DU CONGRÈS}

Hôtel Sofitel Lyon-Bellecour 20 Quai Gailleton - 69002 Lyon

Téléphone : 0472412020 - Fax : 0472400550

\section{ACCUEIL DU CONGRÈs}

Hôtel Sofitel Lyon-Bellecour à partir de 8 h30

\section{ACCÈS AU LIEU DU CONGRÈS}

L'hôtel Sofitel Lyon-Bellecour est situé au centre ville, près de la Place Bellecour, sur la rive droite du Rhône

\section{RÉDUCTION TRANSPORT}

Air France et SNCF Envoi des Imprimés permettant de bénéficier d'une réduction sur demande 


\title{
DROIT D'INSCRIPTION
}

- INSCRIPTION À LA TOTALITÉ DU CONGRÈS (REPAS DE MIDI COMPRIS)

\author{
avant le 01/11/98 \\ Membre de la SALF \\ $1100 \mathrm{FF}$ \\ après le 01.11 .98 \\ $1300 \mathrm{FF}$ \\ Non membre de la SALF \\ $1500 \mathrm{FF}$ \\ $1700 \mathrm{FF}$ \\ Interne, Doctorant, Paramédical \\ $700 \mathrm{FF}$ \\ $700 \mathrm{FF}$ \\ -Inscription à une seule journée du congrès (repas de midi compris) \\ soit le $10 / 12$, soit le $11 / 12$, soit le 12/12/1998 \\ $500 \mathrm{FF}$ \\ - Inscription au repas de gala (vendredi 11/12/1998) $\quad 500 \mathrm{FF}$

\section{HÉBERGEMENT} \\ (prix à titre indicatif, par nuit, petit déjeuner \& taxe de séjour non compris)
}

\section{Hôtel Sofitel Lyon-Bellecour *****}

chambre simple : $\quad 800 \mathrm{FF}$ chambre double : $850 \mathrm{FF}$

Hôtels proches du lieu du congrès

$* *$

$* * *$

$* * * * * *$

Chambre simple

250-305 FF

300-400 FF

$580 \mathrm{FF}$

Chambre double

305-370 FF

$330-480 \mathrm{FF}$

$630 \mathrm{FF}$

\section{AnNulation}

Pour être recevable, toute annulation doit être faite par fax ou par courrier avant le 27 /11/1998.

Une somme de $300 \mathrm{FF}$ sera retenue pour frais administratifs. Au-delà de cette date aucun remboursement ne pourra être effectué. 